\title{
Experimental And Numerical Study On Surface Generated Mechanism of Robotic Belt Grinding Process Considering The Dynamic Deformation of Elastic Contact Wheel
}

\author{
Mingjun Liu \\ Northeastern University \\ Yadong Gong ( $\square$ ydgong@mail.neu.edu.cn ) \\ Northeastern University \\ Jingyu Sun \\ Northeastern University \\ Yuxin Zhao \\ Northeastern University \\ Yao Sun \\ Northeastern University
}

\section{Research Article}

Keywords: Surface generated mechanism, Robotic belt grinding, Elastic contact, Dynamic deformation

Posted Date: October 26th, 2021

DOI: https://doi.org/10.21203/rs.3.rs-1004428/v1

License: (c) (1) This work is licensed under a Creative Commons Attribution 4.0 International License. Read Full License

Version of Record: A version of this preprint was published at The International Journal of Advanced Manufacturing Technology on April 16th, 2022. See the published version at https://doi.org/10.1007/s00170-022-09067-6. 
[Title Page]

\section{Article Title}

Experimental and numerical study on surface generated mechanism of robotic belt grinding process considering the dynamic deformation of elastic contact

$$
\text { wheel }
$$

Authors

Mingjun Liu, Yadong Gong*, Jingyu Sun, Yuxin Zhao, Yao Sun

Author affiliations: Northeastern University, Shenyang, China

Correspondence information:

Correspondence author name: Y.D. Gong

Affiliation: Northeastern University, Shenyang, China

Department address: School of Mechanical Engineering and Automation:

Northeastern University, Shenyang, P.R.China 110819

Email address: gongyd@mail.neu.edu.cn

Telephone number: 86-139-4051-8488 


\title{
Experimental and numerical study on surface generated mechanism of robotic belt grinding process considering the dynamic deformation of elastic contact wheel
}

\begin{abstract}
In the robotic belt grinding process, the elastic contact condition between the flexible tool and the workpiece is a critical issue which extremely influences the surface quality of the manufactured part. The existing analysis of elastic removal mechanism is based on the statistic contact condition but ignoring the dynamic removal phenomenon. In this paper, we discussed the dynamic contact pressure distribution caused by the non-unique removal depth in the grinding process. Based on the analysis of the equivalent removal depth of a single grit and the trajectories of grits in manufacturing procedure, an elastic grinding surface topography model was established with the consideration of the dynamic contact condition in the removing process. Robotic belt grinding experiments were accomplished to validate the precision of this model, while the result showed that the surface roughness prediction error could be confined to $11.6 \%$, which meant this model provided higher accuracy than the traditional predicting methods.
\end{abstract}

Keywords: Surface generated mechanism; Robotic belt grinding; Elastic contact; Dynamic deformation $a_{s}$, the length of the contact area in the static contact condition.

$a_{d}$, the length of the contact area in the dynamic contact condition.

$a_{p, \text { total }}$, the totally removal depth.

$a_{p, g r i t}(x)$, the removal depth of a single grit.

$\bar{a}_{p, g r i t}(x)$, the equivalent removal depth of a single grit.

$\bar{a}_{p, \text { finial }}(x)$, the equivalent removal depth of final single grit.

$b_{m n}$, the offset of grain trajectory relative to the contact area center point.

$B_{w}$, the width of the workpiece.

$B_{c}$, the width of the contact wheel.

$B$, the width of the contact area.

$E_{R u b}$, the Young's modulus of the contact wheel rubber outer layer.

$E_{A l}$, the Young's modulus of the contact wheel aluminum inner core.

$E_{1}$, the Young's modulus of the contact wheel.

$E_{2}$, the Young's modulus of the workpiece.

$E^{*}$, the equivalent Young's modulus.

$e_{R a}$, the arithmetic average deviation error.

$e_{R t}$, the maximum altitude error .

$f_{0}$, the frequency of the belt machine tool driving wheel.

$F_{\text {grit }}(x)$, the normal force of a single grain.

$F_{n}$, the normal force between the workpiece and the contact wheel.

$h_{g}$, the height of a single grain.

$h_{s}$, the height of the gullies on grinded surface.

$h_{d}(x)$, the workpiece contour approximate equation in the contact area.

HV, the Vicker's hardness of the workpiece.

$H_{u}$, the ungrinded surface height.

$H_{g}$, the grinded surface height.

$k_{m}$, the coefficient of grit morphology.

$k_{p}$, the coefficient of material removal.

$k_{w}$, the coefficient of grit wear. 
$k_{h}$, the coefficient of surface topography.

$k_{R a}$, the coefficient of arithmetic average deviation value caused by the stacking removal.

$k_{R t}$, the coefficient of maximum altitude value caused by the stacking removal.

$p_{s}(x, y)$, the contact area pressure distribution in static contact condition.

$p_{d}(x, y)$, the contact area pressure distribution in dynamic contact condition.

$R$, the radius of the contact wheel.

$r$, the radius of the contact wheel inner core.

$R_{0}$, the radius of the driving wheel.

$R_{a, e x p}$, the arithmetic average deviation value of the profile from experiments.

$R_{t, e x p}$, the maximum altitude value of the profile from experiments.

$R_{a, i d e}$, the ideal arithmetic average deviation value of the profile.

$R_{t, i d e}$, the ideal maximum altitude value of contour.

$R_{a, p r e}$, the arithmetic average deviation value of the profile from the prediction model.

$R_{t, p r e}$, the maximum altitude value of contour from the prediction model.

$S_{\text {cont }}$, the area of wheel-workpiece contact space.

$T$, the type of grain size.

$v_{s}$, the linear velocity of the belt.

$v_{f}$, the robotic feed speed.

$v_{m}$, the equivalent cutting speed.

$v_{l}$, the Poisson ratio of the contact wheel.

$v_{2}$, the Poisson ratio of the workpiece..

$\delta_{\max }$, the maximum deformation value of the contact wheel in the contact area.

$\delta_{s}(x, y)$, the contact area deformation in static contact condition.

$\delta_{d}(x, y)$, the contact area deformation in dynamic contact condition.

$\theta$, the .apex angle of the grains

$\psi$, the angle between robotic feeding speed orientation and cutting velocity orientation

$\varphi$, the angle between grains arrangement orientation and robotic feed orientation.

$\Delta l_{s}$, the width of the gullies on grinded surface

$\Delta l_{x}$, the horizontal spacing of grains

$\Delta l_{y}$, the vertical spacing of grains.

\section{Introduction}

The developments of advanced manufacturing industries, such as aerospace, new energy power generation, new energy vehicles, promote the demands of manufacturing components with free-form surfaces, just as engine blades, fan blades, wheel hubs and new energy vehicle bodies. In contemporary industrial productions, artificial works and special machine tools are the main craft for the preparation of parts with curved surfaces. These products fabricated by the manual grinding are sick in the rate of producing and qualities among different batches. While, manufacturing with special machine tools also takes disadvantages like the cost of equipment procurements. Robot assisted abrasive belt grinding is one species of free-form surface fabricating method with enormous potentials. Because of its structural characteristics, the stiffness of the serial robots is much lower than the one of the machine tools. While the elastic contact wheels of belt machine tools can provide passive flexibility which can reduce the deformation of robots in machining process. Nevertheless, the elastic deformation of the contact wheel complicates the grain trajectories in the removal process. The removal process and surface forming mechanism of robot assisted belt grinding is widely investigated [1-2].

For composite curved surface details with the requirement of dynamic characteristics, like blades, the surface 
roughness is an important property index of the qualities in service conditions. The threshold exceeded surface roughness causes air turbulent at the blade edge, resulting in the reduction of blade dynamic performance, and power losses of engine. Enormous surface roughness may cause cracks or even fracture due to the stress concentration effects, and ruined the performances in high temperature service conditions [3-5].

At present, the universal method for predicting the rigidly grinding surface roughness is based on the undeformed chip thickness model. The maximum undeformed chip thickness (MUCT) is a comprehensive coefficient which integrates both the cutting parameters and the grain status [6-8]. While the MUCT method is unable to predict exact value for elastic contact grinding procedures, such as belt grinding. Though the error can be reduced by introducing some elastic contact coefficients, the MUCT method is deficient in theoretical basis and the trend of MUCT predicted values is inconsistent with the experimental values [9].

Therefore, it is profitable to intensively investigate the removal mechanism of the elastic contact between abrasive grains and workpiece surfaces in elastic grinding process, which includes scratching, ploughing and cutting steps. Zhu et al. evaluated the mechanism of robotic belt grinding by the perspective of grinding force [10]. The accomplishment of their work is the settlement of the micro cutting force model compensated with sliding, ploughing and cutting components. Based on the force model, the influence of force components on the machined surface roughness is discussed. Yang et al. instituted an energy efficiency model based on the friction coefficient model of a single spherical grain from the perspective of abrasive geometry [11], which showed that the ploughing energy took more proportion than the cutting energy and the scratching energy, while the grinding depth of a single particle was much smaller than the radius of grains. Based on the mechanism evaluation of robotic belt grinding, a noval grinding force model is accomplished by $\mathrm{Xu}$ et al. to predict the grinding removal depth and profit a quantificational machining process of robotic belt grinding [12,13]. Agustina and Segreto appraised the surface roughness obtained by robot-assisted polishing experiments with the analysis of the acoustic emission signal frequency domain features [14,15]. Zou et al. established a surface quality model considering the no-linear characteristic of rubber [16]. Pamdiyan et al. proposed an on-line multi-sensor integration system, which was composed with force sensors, acceleration sensors and acoustic emission sensors, to predict the surface roughness formed by robotic belt grinding through the neural network method [17].

In this paper, a noval surface topography model for surface roughness prediction in robotic belt grinding procedure considering the dynamic deformation of the elastic contact wheel is provided. With the infrastructures of the elastic plane-cylinder contact model and Preston equation, an original distribution of the contact pressure considering the dynamically removal volume during the elastic grinding procedure is attained. According to the plastic contact theory, the motional removal depth of a single pyramidal grain is gained by its relationship with the normal force of the grain. On the basis of the study in the equivalent removal depth of a single grit and the trajectories of grits in elastic grinding procedure an ideal surface topography model considering the effects of grain sizes is accomplished. Based on the topography model, the surface roughness obtained by robotic belt grinding can be predicted by introducing coefficients which means the effects of stacking removal phenomena. Through the robotic belt grinding experiments, the accuracy of the roughness predicting value is proved by comparing with the values in experiments.

\section{The dynamic model and surface topography model involved in robotic belt grinding}

The workpiece settled on the actuator at the end of the robot is grinded by the belt grinding machine tool. During the grinding procedure, caused by the contact force between the contact wheel and the workpiece, deformations happen on both of the contact wheel and the workpiece while the one of the contact wheel is greater. Since getting into the contact area, normally, the grains adhered on the belt suffer three steps: the scratching step, the ploughing step and the cutting step. 


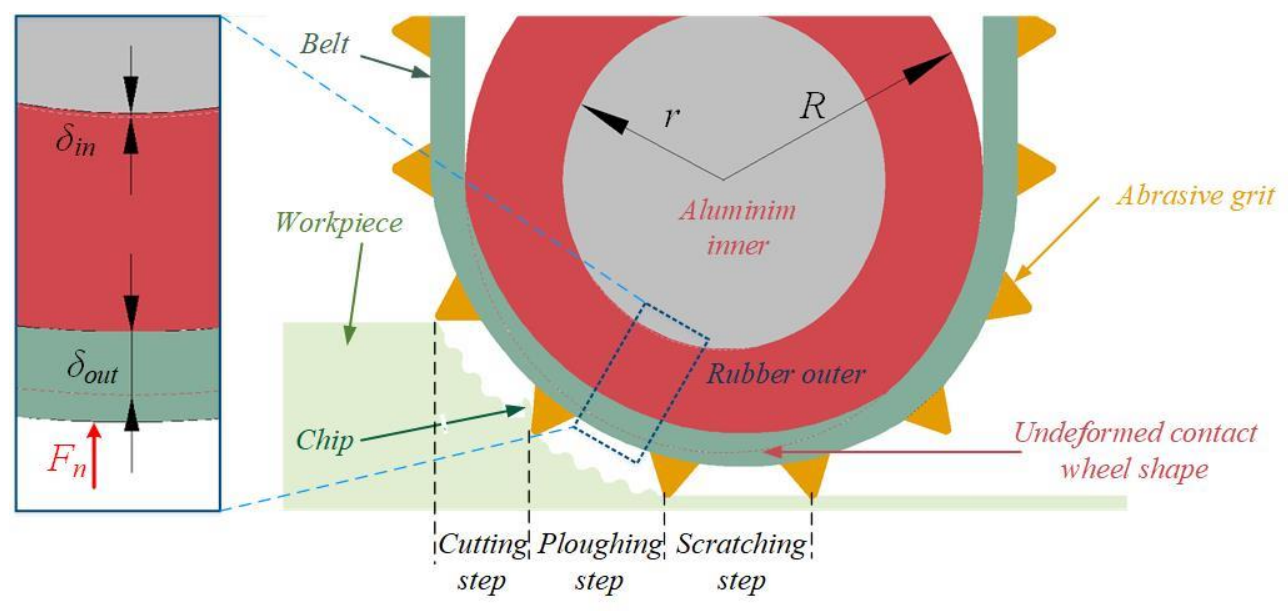

Figure1. The removing mechanism of the robotic belt grinding

\subsection{The dynamic model of contact pressure}

The normal force which generates the material removal is administered by the contact between the workpiece and the contact wheel. The contact wheel is mainly composed of an aluminum inner core and a rubber outer layer. In the research of the contact state, the deformation of the abrasive belt is ignored as for its thickness is much smaller than the radius of the contact wheel, while the diagrammatic sketch of the status of the contact interfaces is shown in Fig.1, which means both of the aluminum inner core and rubber outer layer are compressed under stress [9]. Therefore, the composite Young's modulus of the contact wheel is crucial in establishing the contact model between the workpiece and the contact wheel. The combined Young's modulus of contact wheel with considering the deformations of the inner and the outer is shown as Eq.1 and Eq.2.

$$
E_{1}=\frac{r}{\frac{r}{E_{A l}}+\frac{r}{R} \frac{R-r}{E_{R u b}}}
$$

$\delta_{c}=\delta_{\text {in }}+\delta_{\text {out }}=\frac{F_{n} r}{S_{\text {cont }} E_{A l}}+\frac{F_{n} r(R-r)}{r S_{\text {cont }} E_{\text {Rub }}}(2)$

In the robotic abrasive belt grinding procedure, both of the flexible contact wheel and the workpiece elastic take deformation. Though the elastic characteristic of rubber is non-linear, as for the elastic deformation is much smaller than the thickness of the outer layer, the linear elastic model can be used to calculate the rubber deformation. It is assumed that there is no relative motion between the wheel and the workpiece, which means that the contact is static. The contact wheel is abstracted as an elastic cylinder, while the workpiece is simplified as an elastic plane. According to the elastic cylinder-plane contact model [18], the normal contact force $F_{n}$ is shown as Eq.3.

$F_{n}=\frac{\pi}{4} E^{*} B \delta_{\max }, B=\min \left(B_{w}, B_{c}\right)(3)$

The equivalent Young's modulus $E^{*}$ is shown in Eq.4.

$\frac{1}{E^{*}}=\frac{1-v_{1}^{2}}{E_{1}}+\frac{1-v_{2}^{2}}{E_{2}}$

The length of the static contact area between the workpiece and the contact wheel is expressed as Eq.5.

$$
a_{s}=2 \sqrt{R \delta_{\text {max }}}
$$


The static contact area express as Eq.6.

$S_{\text {cont }}=2 B \sqrt{R \delta_{\text {max }}}(6)$

The static pressure $p_{s}(x, y)$ at an arbitrary point $P(x, y)$ in the contact area is shown in Eq.7.

$p_{\mathrm{s}}(x, y)=\sqrt{\frac{E^{*} F_{n}}{\pi B R}\left(1-\left(\frac{x}{a_{s}}\right)^{\frac{1}{2}}\right)}$

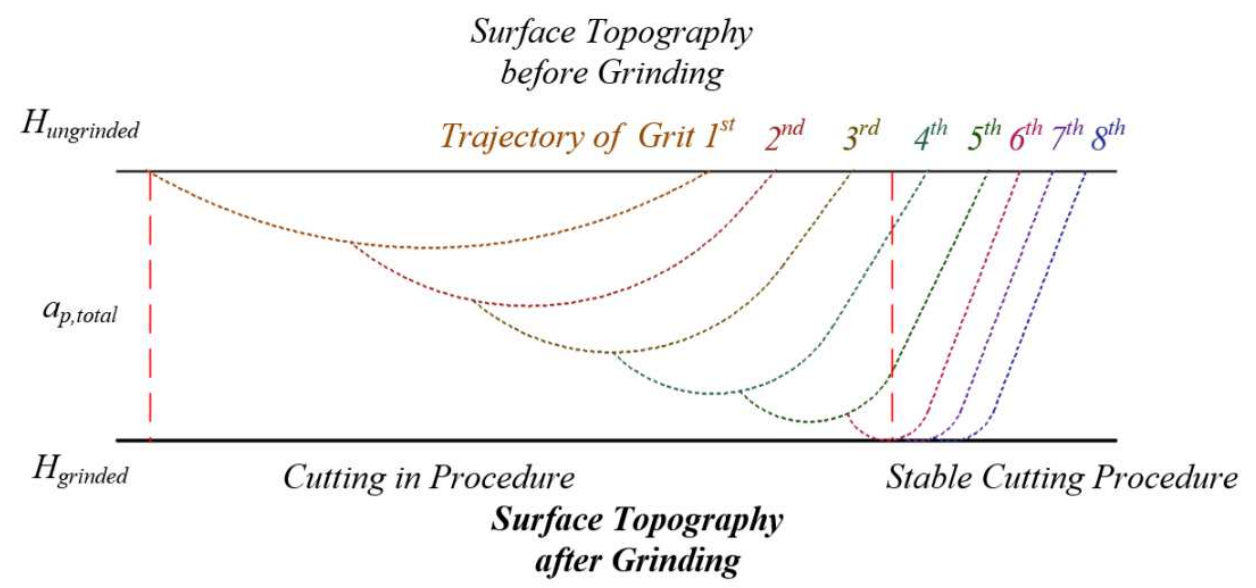

Figure 2. The formation of contact area

The deformation at $\mathrm{P}(x, y)$ in the contact area is shown in Eq.8.

$\delta_{s}(x, y)=\delta_{\max } \sqrt{\left(1-\left(\frac{x}{a_{s}}\right)^{\frac{1}{2}}\right)}$

At present, the static contact models, such as Hertz contact model or elastic cylinder-plane contact model, are generally used to predict the material removal volume in robotic belt grinding. The static contact models suppose that the contact parameters, such as contact area and contact stress distribution, are independent with material removal volume in grinding process. However, in the actual manufacturing, due to the grinding removal effect of the adhesive grits, the actual contact surface between the wheel and the workpiece is not an ideal horizontal plane. As Fig. 2 shows, the leading edge of the actual contact area is the ungrinded surface, while the training edge is the grinded surface. The height of workpiece in contact area is analyzed. In the coordinate system of the contact area $O X Y Z$, which the orientation of $x$ axis is the robotic feed direction, $y$ axis is perpendicular to $x$ axis and plane $X O Y$ is parallel to ungrinded workpiece surface, $z$ axis is perpendicular to $x$ and $y$ axis.

$$
\left\{\begin{array}{l}
\delta_{d}\left(x_{1}\right)=0 \\
\delta_{d}\left(x_{2}\right)=0
\end{array}, x_{1}>x_{2}(9)\right.
$$

As $x=x_{1}$, the height of the workpiece is the ungrinded surface height $H_{u}$, while as $x=x_{2}$, is the grinded surface height $H_{g}$, which is expressed as Eq. 10 .

$$
H_{u}-H_{g}=a_{p, \text { total (10) }}
$$

where, $a_{p, t o t a l}$ is the totally removal depth, which can be obtained by Preston Equation, as Eq.11 [19].

$a_{p, \text { total }}=\frac{k_{p} F_{n} v_{m}}{v_{w}}$ 
In the grinding procedure, the length of the dynamic contact area $a_{d}$ is expressed as Eq.12

$a_{d}=\sqrt{\left(\sqrt{R \delta_{\max }}+\sqrt{R\left(\delta_{\max }-a_{p, \text { total }}\right)}\right)^{2}+a_{p, \text { total }}^{2}} \approx \sqrt{R \delta_{\max }}+\sqrt{R\left(\delta_{\max }-a_{p, \text { total }}\right)}$

The workpiece contour in contact area is fitted with a straight line, as shown in Fig.3, the approximate equation $h_{d}(x)$ is shown as Eq.13,

$h_{d}(x)=\frac{a_{p, t o t a l}}{\left(\sqrt{R \delta_{\max }}+\sqrt{R\left(\delta_{\max }-a_{p, \text { total }}\right)}\right.}$

$\cdot\left(x+\sqrt{R\left(\delta_{\max }-a_{p, \text { total }}\right)}-a_{p, \text { total }}\right)$

where, the equivalent cutting speed $v_{m}$ is shown as Eq.14

$v_{m}=v_{s} \pm v_{w}, v_{s}=2 \pi R_{0} f_{0}$

where, $v_{s}$ is the linear velocity of the belt, $v_{w}$ is the feed speed of the robot, '+/-' means that the linear velocity of contact wheel and the workpiece feed speed takes the same/opposite direction.

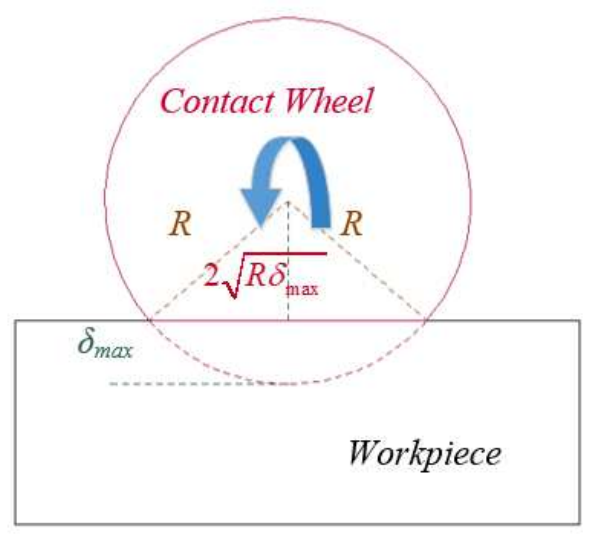

(a) Static contact model

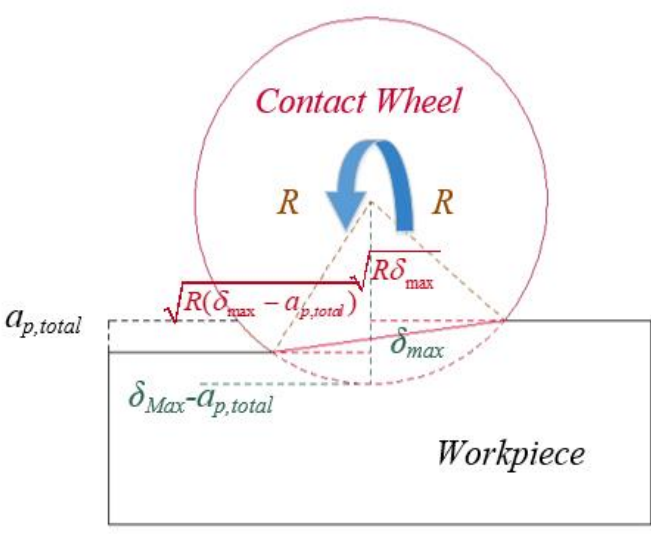

(b) Dynamic contact model

Figure 3. Model of static and dynamic contact condition

Due to the Young's modulus of the workpiece is much larger than that of the contact wheel, it can be regarded as that only the contact wheel takes the elastic deformation, while the deformation of the workpiece is zero, which is shown as Eq. 15 .

$\left\{\begin{array}{c}\delta_{w}=0 \\ \delta_{c}=\delta(x)\end{array}\right.$

The removal volume coefficient $k_{r}(x)$, expressed as Eq.16, indicates the reduction of the contact wheel compression caused by material removal in the stable cutting procedure.

$k_{r}(x)=\frac{\delta_{d}(x)}{\delta_{s}(x)}=\frac{\delta_{s}(x)+h_{d}(x)}{\delta_{s}(x)}(16)$

In the dynamic contact model, the dynamic pressure $p_{d}(x, y)$ at an arbitrary point $P(x, y)$ in the contact area is shown in Eq.17.

$$
p_{d}(x, y)=\frac{\delta_{s}(x)+h_{d}(x)}{\delta_{s}(x)} \sqrt{\frac{E^{*} F_{n}}{\pi B R}\left(1-\left(\frac{x}{a_{s}}\right)^{\frac{1}{2}}\right)}
$$

\subsection{The single grain grinding depth and material removal volume}

The abrasive particles could be abstracted as pyramids. As Fig. 4 displays, the characteristics of the abrasive 
particle morphology, such as the height of abrasive grits $h_{\text {grit }}$, the horizontal spacing between two adjacent abrasive grits is $\Delta l_{x}$, vertical spacing $\Delta l_{y}$, are attained. The angle between the relative surfaces $\theta$ is shown in Eq.18.

$\tan \frac{\theta}{2}=\frac{\Delta l_{x}}{2 h_{g}}(18)$

The abrasive particles trajectory equation on the contact area plane $X O Y$ is shown as Eq.19 [20].

$y=\tan \psi \cdot x+b_{m n}$

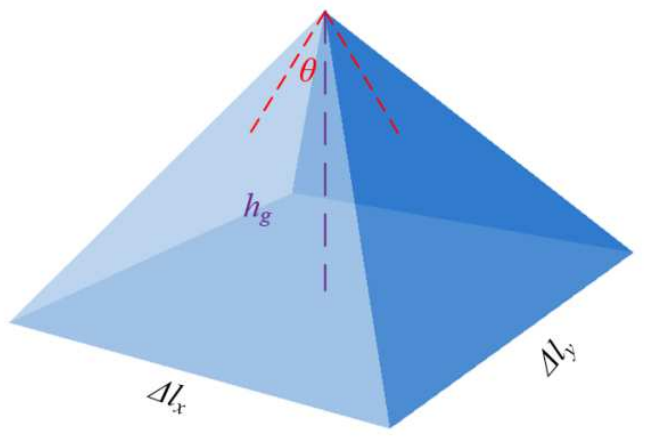

Figure 4. The grit morphology characteristics

where, $\psi$ is the angle between the robotic feeding orientation and the cutting velocity orientation, $b_{m n}$ is the offset of grain trajectory relative to the contact area center point.

The normal force of a single grit $F_{\text {grit }}(x)$ is expressed as Eq.20.

$F_{\text {grit }}(x)=\int_{x-\frac{1}{2} \Delta l_{x}}^{x+\frac{1}{2} \Delta l_{x}+\frac{1}{2} \Delta l_{y}} \int_{\frac{1}{2} \Delta l_{y}} p_{d}(x) d y d x$

Wang used the material hardness to calculate the removal depth of a single grain [21].Zhang discovered that the grain wear condition influenced material removal depth[22]. Asikuzun provided the relationship between Vickers hardness, normal force and indentation depth [23]. As the grain morphology is similar with the Vickers hardness testing penetrator, the removal depth of a single grain $a_{p, g r i t}(x)$ can be calculated as Eq.21.

$a_{p, \text { grit }}(x)=k_{m} k_{w} \sqrt{\frac{F_{\text {grit }}(x)}{\mathrm{Hv} \cdot \tan ^{2} \frac{\theta}{2}}}(21)$

where, $k_{w}$ is the coefficient of grain wear. $k_{m}$ is the coefficient of abrasive grit morphology as Eq.22 shown.

$k_{m}==0.9032 \theta-0.9594$

When the pressure is less than 0.4HB, only elastic deformation occurs [24]. As Eq.21 shows when the grains are abstracts as ideal pyramids, the pressure between the grain and the workpiece is $p_{\text {grit }}=\mathrm{HV}>0.4 \mathrm{HB}$, so in this model, there is no scratching process.

\subsection{The surface topography model}

The removal width of a single grit is much smaller than the horizontal distance between two adjacent abrasive grits $\Delta l_{x}$. Actually, the morphology of workpiece surface is formed by the grinding tracks of different grains at different times. In order to express the vertical positions of different grains reaching the cross section, the 
equivalent cutting depth of single abrasive particles is introduced as Eq.23.

$\bar{a}_{p, g r i t}(x)=\frac{S_{p, g r i t}(x)}{\Delta l_{x}}=\frac{a_{p, g r i t}^{2}(x)}{2 \Delta l_{x} \cot \left(\frac{\theta}{2}\right)}(23)$

Since the removal depth of single grit is much smaller than the elastic contact deformation, the equivalent removal depth of a single abrasive grain $\bar{a}_{p, i}(x)$, shown as Eq.24, can be regarded as a constant volume in the calculation of the robotic belt grinding surface roughness.

$$
\bar{a}_{p, \text { finial }}(x) \approx \frac{a_{p, N q}^{2}(x)}{2 \Delta l_{x} \cot \left(\frac{\theta}{2}\right)}(24)
$$

As for the angle $\varphi$ between the grain arrangement direction and the robot feed direction, the horizontal distance between the locations which are trajectories of two adjacent grains in the same row in cross section is $\Delta l_{y} \times \sin (\varphi)$, while the vertical one is $-\bar{a}_{p, \text { finial }}(x)$. The points in the area of $\left\{\left(x_{\text {section }}, y_{\text {section }}\right) \mid 0<x_{\text {section }} \leq \Delta l_{x},-a_{p, N(x)}<y_{\text {section }} \leq 0\right\}$ can be expressed with $\left(x_{m}, y_{m}\right)$ as Eq.5

$$
\begin{aligned}
& \left\{\begin{array}{c}
x_{m}=\operatorname{rem}\left(m \cdot \sin (\varphi) \Delta l_{y}, \Delta l_{x}\right) \\
y_{m}=-m \cdot \bar{a}_{p, \text { finial }}(x)
\end{array},\right. \\
& m=1,2 \ldots,\left\lfloor\frac{2 \Delta l_{x} \cot \left(\frac{\theta}{2}\right)}{a_{p, N q}(x)}\right\rfloor
\end{aligned}
$$

where, $\operatorname{Rem}()$ is the remainder function.

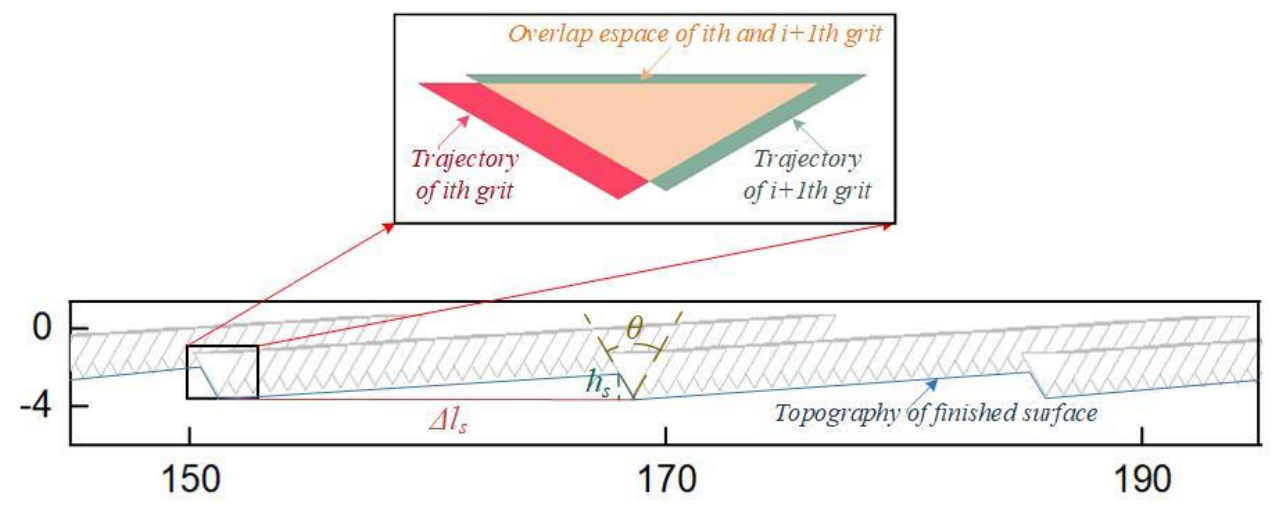

Figure 5. The ideal surface topography formed by robotic belt grinding

The surface formed by the grain tracks are shown as Fig.5. The grinded surface morphology, which is the blue line in Fig.6, is the envelope of abrasive trajectories. $\Delta l_{s}$ is the width of the gullies on grinded surface, while $h_{s}$ is the height, expressed as Eq.26.

$h_{s}=k_{h} a_{p, N q}(0)(26)$

where, $k_{h}$ is the surface topography coefficient.

The grinding surface is the envelope of abrasive trajectories. Through the definitions of the arithmetic average 
deviation value of the profile $R_{a}$ and the maximum altitude value of contour $R_{t}$, calculated as Eq.27.

$\left\{\begin{array}{c}R_{a}=\frac{1}{l} \int_{0}^{l} y d x \\ R_{t}=y_{\max }-y_{\min }\end{array}\right.$

The ideal surface roughness along the robot feed direction of belt grinding can be obtained as Eq.28.

$\left\{\begin{aligned} R_{a, i d e} & =\frac{h_{s}}{4}=\frac{k_{h} a_{p, N q}(x)}{4} \\ R_{t, \text { ide }} & =h_{s}=k_{h} a_{p, N q}(x)\end{aligned}\right.$

In the surface profile model establishing process, the relationship between different trajectories of grits is assumed to be the parallel removal mode, which means that the adjacent trajectory is approximately horizontally arranged. The adjacent removal mode is shown in Fig.6. Nevertheless, in the actual surface forming process, besides the adjacent removal mode, the stacking removal mode is also exist. The stacking mode means that the adjacent grinding tracks overlap in the vertical direction, therefore the grinding depth stacking one by one, which makes the gully depth on the surface profile exceeding the ideal one $h_{s}$. The stacking removal mode is shown in Fig.6.

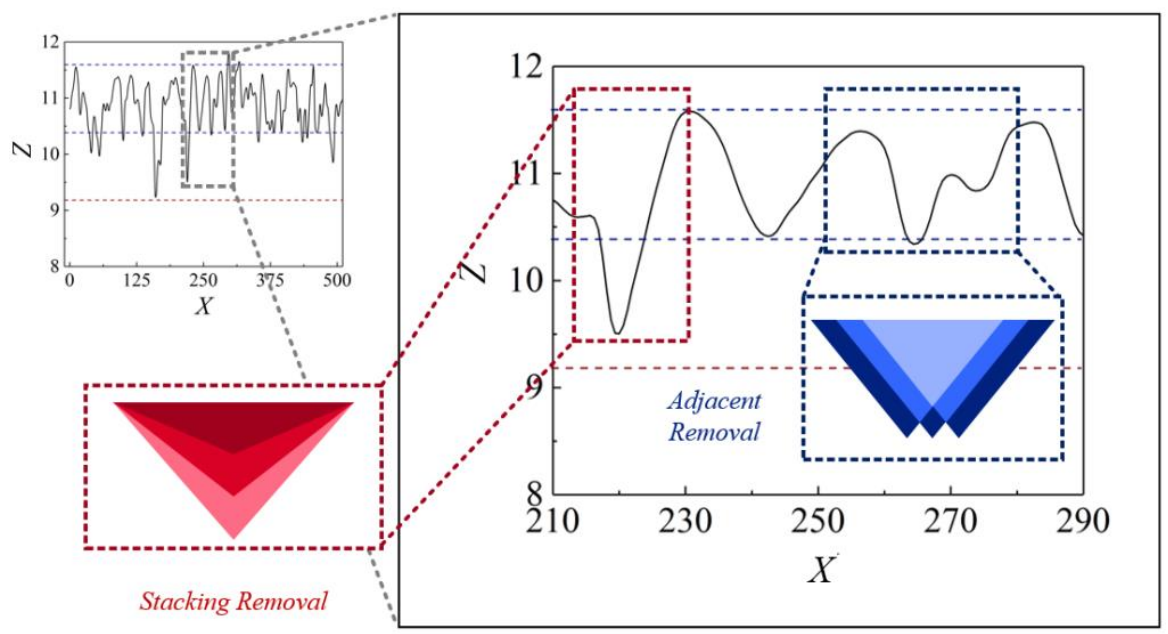

Figure 6 . The adjacent removal mode and the stacking removal mode

Through the analysis of the robotic belt grinding surface topography, it can be found that the adjacent removal mode is the main stream of material removing. Contrarily, the stacking removal mode occurs randomly, while the maximum removal depth formed by the stacking removal mode has a positive correlation with the single grit removal depth.

Above all, the robotic belt grinding surface roughness of can be predicted as Eq.29,

$$
\left\{\begin{array}{c}
R_{a, p r e}=k_{R a} R_{a, i d e}=\frac{k_{h} k_{R a} a_{p, N q}(x)}{4} \\
R_{t, p r e}=k_{R t} R_{t, i d \mathrm{e}}=k_{h} k_{R t} a_{p, N q}(x)
\end{array}\right.
$$

where, $k_{R a}$ is the coefficient of arithmetic average deviation value caused by the stacking removal, $k_{R t}$ is the one of maximum altitude value. 


\section{Experiments}

\subsection{Experimental conditions}

All the robotic belt grinding experiments were conducted on the robotic belt grinding system mainly composed of a 6-DOF robot with the type of Motorman DX100, as shown in Fig.7. The Inconel 718 superalloy samples which were cut to $500 \mathrm{~mm}$ (length) $\times 15 \mathrm{~mm}$ (width) $\times 10 \mathrm{~mm}$ (thickness) pieces were fixed with the specific fixtures. The belt 237AA (3M Company), covered with $\mathrm{Al}_{2} \mathrm{O}_{3}$ grains, were used in the experiments. The micro-morphologies of the grains were shown by KEYENCE VHX-1000C digital microscope as Fig.8, which verified the grain morphology model.

Table 1. Grains morphology characteristics

\begin{tabular}{llll}
\hline Type of grain size $T(\#)$ & Grain height $h_{g}($ um $)$ & Grains vertical spacing $\Delta l_{x}(u m)$ & Grains horizontal spacing $\Delta l_{y}(u m)$ \\
\hline 30 & 247 & 392 & 411 \\
45 & 291 & 467 & 450 \\
65 & 272 & 508 & 472 \\
80 & 296 & 586 & 526 \\
100 & 389 & 713 & 680 \\
\hline
\end{tabular}

Table 2. Grinding system characteristics

\begin{tabular}{ll}
\hline Items & Conditions \\
\hline \multirow{2}{*}{ Contact Wheel } & $r=50 \mathrm{~mm}, R=100 \mathrm{~mm}, R_{0}=100 \mathrm{~mm}, E_{A l}=70 G p a, E_{R u b}=7.8 \mathrm{Mpa}, v_{l}=0.47, B_{c}=50 \mathrm{~mm}, \psi=0^{\circ}$, \\
& $\varphi=14^{\circ}, b_{m n}=0$ \\
Workpiece & $E_{2}=199.9 \mathrm{GPa}, v_{2}=0.3, H v=3.376 \mathrm{Gpa}, B_{w}=50 \mathrm{~mm}, k_{w}=1.8, k_{R a}=1, k_{R t}=2.1$ \\
\hline
\end{tabular}

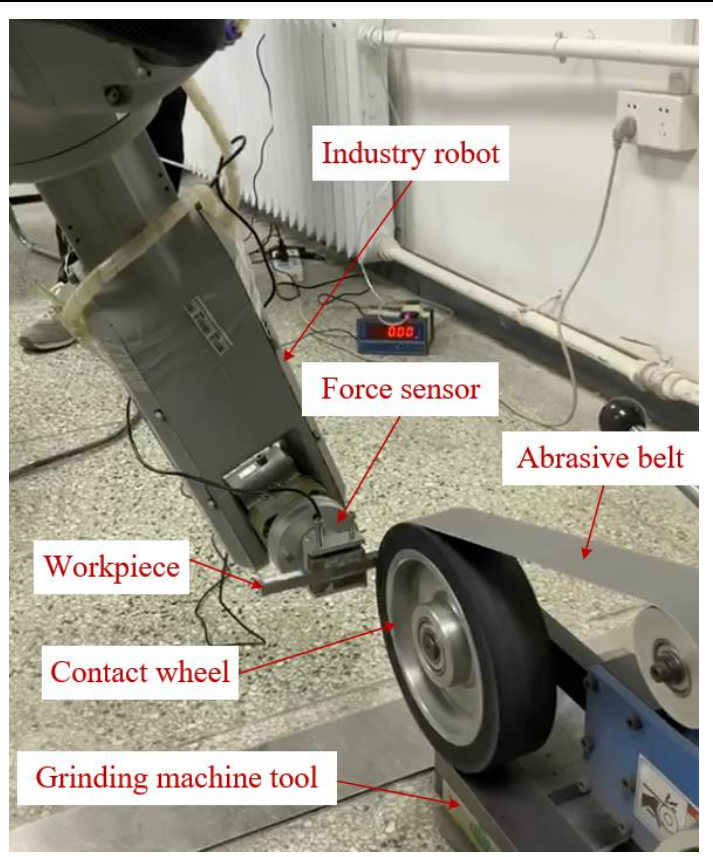

Figure 7.The robotic belt grinding system

The abrasive grit morphology characteristics of each type of abrasive belts are shown in Tab.1.

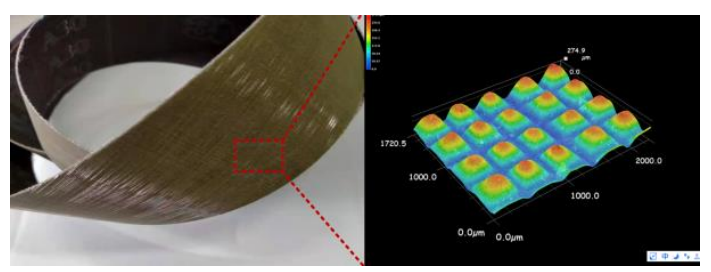

(a) The abrasive grain micro-morphology 


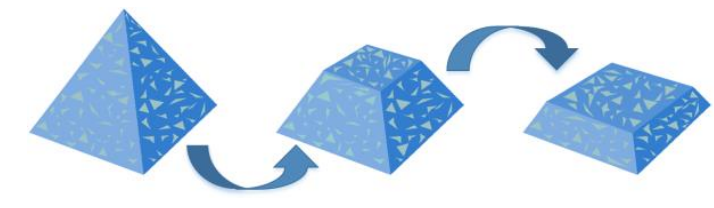

(b) The different wear condition grain morphology model

Figure 8. The morphologies of abrasive grits

The grinding system characteristics in the belt grinding surface roughness model are shown in Tab.2.

\subsection{Experimental design}

The experimental parameters were selected as Tab.3 After the tests, the surface morphologies of the workpieces were scanned by the laser confocal microscope, which could calculated the surface roughness value obtained by robotic belt grinding.

Table 3. Grinding parameters of experiments

\begin{tabular}{ll}
\hline Grinding parameters & Value ranges \\
\hline Type of grain size $T(\#)$ & $30,45,65,80,100$ \\
Driving wheel frequency $f_{0}(\mathrm{~Hz})$ & $5,10,15,20,25$ \\
Robotic feed speed $v_{w}(\mathrm{~mm} / \mathrm{s})$ & $1,3,5,7,9$ \\
Maximum contact depth $\delta_{\max }(\mu \mathrm{m})$ & $20,40,60,80,100$ \\
\hline
\end{tabular}

\section{Verification and discussion}

For the sake of verifying the belt grinding surface roughness mode, Eq.29 was used to calculate the surface roughness predicted value which are compared with the experimental values to characterize the accuracy of the surface roughness model. The arithmetic average deviation error $e_{R a}$ is $11.6 \%$ while the maximum altitude error $e_{R t}$ is $7.03 \%$ respectively, which are calculated as Eq.22 and Eq.2

Eq.23.Through error analysis, the efficiency of the surface roughness model is verified. Compared with the normal static contact surface roughness model, the improved dynamic contact surface roughness model provides higher precisions.

$e_{R a}=\frac{\sum_{i=1}^{i=n_{\text {exp }}} \frac{\left|R_{a, \exp }-R_{a, p r e}\right|}{R_{a, \exp }}}{n_{\exp }} \times 100 \%$

$e_{R t}=\frac{\sum_{i=1}^{i=n_{\text {exp }}} \frac{\left|R_{t, \exp }-R_{t, p r e}\right|}{R_{t, \text { exp }}}}{n_{\text {exp }}} \times 100 \%(31)$

Fig.9 shows the relationships between the surface roughness and the grinding parameters. As shown in Fig.9(a), when the belt linear velocity is under a threshold, the surface roughness model provides an accurate prediction. When the velocity is over the threshold, the surface roughness is much larger than the predicted value. It is caused by the elastic recoil phenomenon of the rubber layer [25].

Both the simulations and the experiments suggest that the surface roughness get deteriorated with the increasing of contact depth, as Fig.9(b) shown, while the feeding speed only makes a slight effect on surface roughness as Fig.9(c) shown.

It is observed that the grain size and contact depth have great influence on the surface quality as Fig. $9(d)$ shows. The abrasive particle size has the most significant effect on the surface roughness, and the surface roughness reaches over $R_{a} 0.6$ when the type of grain is \#100, while the surface roughness is reduced under $R_{a} 0.3$ with \#30. This conclusion is supported by Gorp AV [26], that the most important factor affecting the workpiece 
roughness is the grain size.

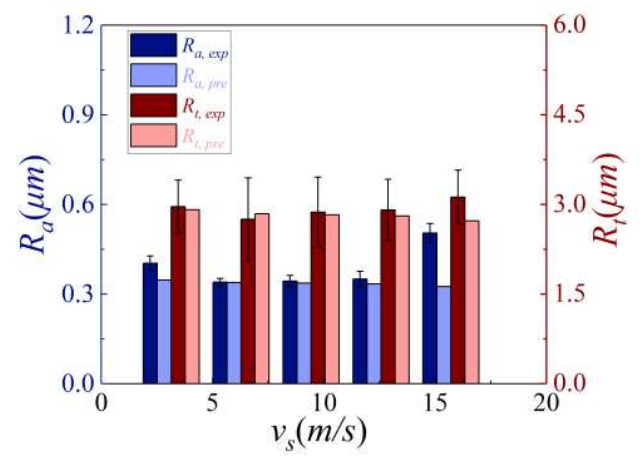

(a)

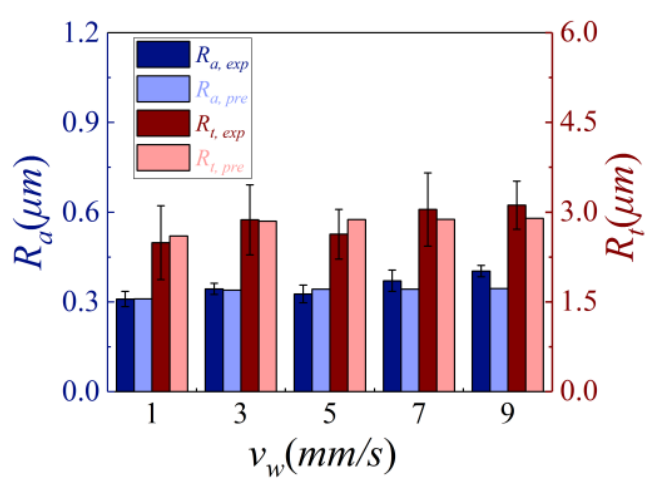

(c)

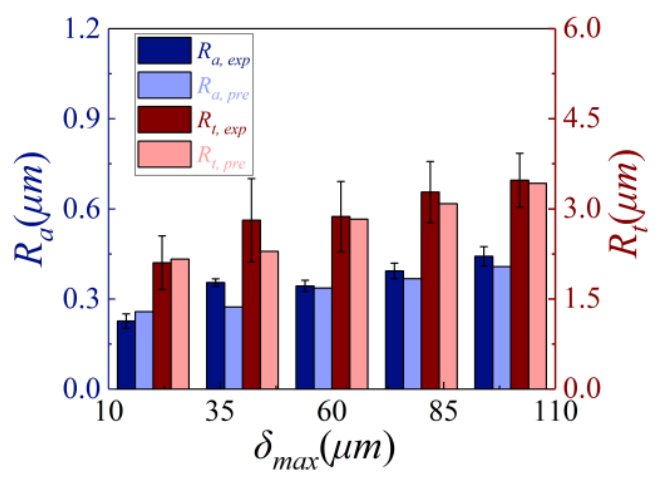

(b)

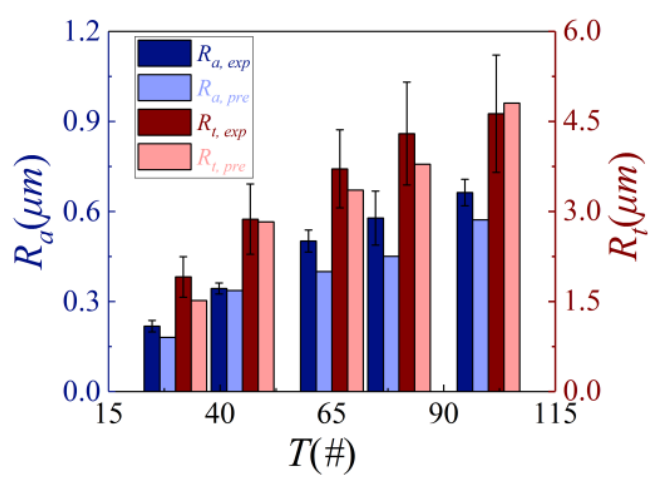

$(d)$

(a)The relationship between the surface roughness and the belt cutting velocity; $(b)$ the relationship between the surface roughness and the maximum contact depth; $(c)$ the relationship between the surface roughness and the robotic feed speed; $(d)$ the relationship between the surface roughness and the size of grains

\section{Conclusion}

Figure 9. The relationships between the surface roughness and the grinding parameter

In this study, a novel surface topography model of robotic belt grinding procedure considering the dynamic deformation of the elastic contact wheel is proposed. Based on the elastic plane-cylinder contact model and Preston equation, an original distribution of the contact pressure considering the dynamically removal volume during the elastic grinding procedure is attained. Besides, on the basis of the study in the equivalent removal depth of a single grit and the trajectories of grits in elastic grinding procedure, an ideal surface topography model considering the effects of grain sizes is accomplished. The results indicated that the surface topography formed by elastic grinding related to the removal depth of a single grain, unlike the one formed by rigid grinding which is effected by the totally removal depth. Moreover, the surface roughness forecast model is provided by considering the removal depth of a single grain and the trajectory of active grains in robotic belt grinding process, and the verified experimental results of robotic belt grinding disclosed that the prediction error can be confined to $11.6 \%$, which can predict the surface topography information of robotic belt grinding procedure more accurately compared with the traditional predicted models.

\section{Declaration}

Acknowledgments Funding The authors would like to acknowledgement financial support from the National Natural Science Foundation of China Key (Joint Fund) Project (no. U1908230)

Conflicts of interest/Competing interests The authors declare no competing interests. 
Availability of data and material The datasets used or analyzed during the current study are available from the corresponding author on reasonable request.

Code availability Not applicable.

Ethics approval The authors state that the present work is in compliance with the ethical standards.

Consent to participate Not applicable.

Consent for publication All authors agree to publish.

Authors' contributions Mingjun Liu: Investigation, Conceptualization, Methodology, Experiment, Writing original draft. Yadong Gong: Funding and acquisition, reviewed \& edited the manuscript, Supervision. Jingyu Sun: Investigation, Experiment. Yuxin Zhao: Investigation, Experiment. Yao Sun: Supervision.

\section{References}

[1]Zhu D, Feng X, Xu X (2020) Robotic grinding of complex components: A step towards efficient and intelligent machining - challenges, solutions, and applications[J]. Robot. Cim-Int. Manuf. 65:101908. https://doi.org/10.1016/ j.rcim. 2019.101908

[2]Lee J (1985) Developing an end-of-arm tooling for robotic grinding machining application. Int J Adv Manuf Technol, 1985, 1(1):27-36. https://doi.org/10.1007/ bf02601580

[3]Qiu L, Qi L, Liu L, Zhang Z, Xu J (2020) The blade surface performance and its robotic machining. Int J Adv Robot Syst 17(2):172988142091409 https://doi.org/10.1177/1729881420 914090

[4]Howell RJ, Roman KM (2016) Loss reduction on ultra high lift low-pressure turbine blades using selective roughness and wake unsteadiness. Aero J 111(1118):257-266. https://doi.org/ 10. 1017/S0001924000004504

[5]Walker JM, Flack KA, Lust EE (2014) Experimental and numerical studies of blade roughness and fouling on marine current turbine performance. Renewable Energy. 66:257-267. https://doi.org/10.10 16/j.renene.2013.12.012 [6]Zhang ZY, Wang B, Kang RK, Zhang B, Guo DM (2015) Changes in surface layer of silicon wafers from diamond scratching. CIRP Ann Manuf Technol 64:349-352 https://doi.org/10.1016/j.ci rp.2015.04.005

[7]Shen B, Song B, Cheng L, Lei XL, Sun FH (2014) Optimization on the HFCVD setup for the mass-production of diamond-coated micro-tools based on the FVM temperature simulation. Surf Coat Technol 253:123-131 https://doi.org/10. 1016/j.surfcoat.2014.05.024

[8]Sun Y, Su ZP, Jin LY, Gong YD, (2021) Modelling and analysis of micro-grinding surface generation of hard brittle material machined by micro abrasive tools with helical chip pocket. J Mater Process Tech 297(1):117242 https://doi.org/10.1016/j.jmat protec.2021.117242

[9]Qu C, Lv Y, Yang Z(2019) An improved chip-thickness model for surface roughness prediction in robotic belt grinding considering the elastic state at contact wheel-workpiece interface. Int J Adv Manuf Tech 104:3209-3217. https://doi.org/10.1007/s00170-019-04332-7

[10]Zhu DH, Xu XH, Yang ZY (2018) Analysis and assessment of robotic belt grinding mechanisms by force modeling and force control experiments. Tribol Int 120:93-98 https://doi.org/ 10.1016/j.tribo int.2017.12.043

[11]Yang ZY, Xu XH, Zhu DH (2019) On energetic evaluation of robotic belt grinding mechanisms based on single spherical abrasive grain model. Int J Adv Manuf Technol 104:4539-4548 https://doi.org/10.1007/s00170-019$\underline{04222-y}$

[12]Xu XH, Yang YF, Pan GF (2018) A Robotic Belt Grinding Force Model to Characterize the Grinding Depth with Force Control Technology. International Conference on Intelligent Robotics \& Applications. Springer. Cham https://doi.org/10.1007/978-3-319-97586-3_26

[13]Xu XH, Ye ST, Yang ZY (2021) Analysis and prediction of surface roughness for robotic belt grinding of complex blade considering coexistence of elastic deformation and varying curvature. Sci China Tech Sci. 64:957-970 https://doi.org/10.1007/s11431-020-1712-4

[14]Agustina BD, MM Marín, Teti R(2014) Surface Roughness Evaluation Based on Acoustic Emission Signals in 
Robot Assisted Polishing. Sensors 14(11): 21514-21522. https://doi. org/10.3390/s14 1121514

[15]Segreto T, Karam S, Teti R (2017) Signal processing and pattern recognition for surface roughness assessment in multiple sensor monitoring of robot-assisted polishing. Int J Adv Manuf Technol 90:1023-1033 https://doi.org/10.1007/ s00170-016-9463-X

[16]Zou L, Liu X, Huang Y (2019) A numerical approach to predict the machined surface topography of abrasive belt flexible grinding. Int J Adv Manuf Technol 104(5-8):2961-2970. https://doi. org/10.1007/s00170-019-04032-2

[17]Vigneashwara Pandiyan, Tegoeh Tjahjowidodo, Meena Periya Samy (2016) In-Process Surface Roughness Estimation Model for Compliant Abrasive Belt Machining Process. Procedia CIRP (46):254 - 257. https://doi.org/10.1016/j.procir.2016. 03.126

[18]VL Popov (2010) Contact Mechanics and Friction: Physical Principles and Applications, 1st Edition Springer-Verlag: Berlin Heidelberg

[19]Lv YJ, Peng Z, Qu C (2020) An adaptive trajectory planning algorithm for robotic belt grinding of blade leading and trailing edges based on material removal profile model. Robot Cim-Int Manuf 66:101987 https://doi.org/10.1016/j.rcim.2020.101987

[20]Huai WB, Lin XJ, Shi YY (2020) Geometric characteristic modeling for flexible contact of sanding wheel-polished complex surface. Int J Adv Manuf Technol 110(2) https://doi.org/ 10.1007/s00170-020-05959-7 [21]Wang GL, Wang YQ, Xu ZX (2009) Modeling and analysis of the material removal depth for stone polishing. J Mater Process Technol 209(5):2453- 2463 https://doi.org/ 10.1016/j.jmatprotec. 2008.05.041

[22]Zhang L, Tam HY, Yuan CM (2002) An investigation of material removal in polishing with fixed abrasives. P I Mech Eng B-J Eng 216(1):103-112 https://doi.org/10.1243/09544 05021519591

[23]Asikuzun E, Ozturk O, Cetinkara HA (2012) Vickers hardness measurements and some physical properties of Pr2O3 doped Bi-2212 superconductors. J Mater Sci-Mater El 23(5):1001-1010 https://doi.org/10.1007/s10854 $\underline{-011-0537-0}$

[24]Zhao YW, D.M. Maietta, Chang L (2000) An asperity microcontact model incorporating the transition from elastic deformation to fully plastic flow. J Tribol-T Asme 122:86-93 https://doi.org/ 10.1115/1.555332

[25]Yang ZY, Chu Y, Xu XH (2021) Prediction and analysis of material removal characteristics for robotic belt grinding based on single spherical abrasive grain model. Int J Mech Sci 190 https://doi.org/10.1016/j.ijmecsci.2020.106005

[26]Gorp A V, Bigerelle M, Mansori M E (2015) Effects of working parameters on the surface roughness in belt grinding process: the size-scale estimation influence. Int J Mater Prod Tec 38(1). https://doi.org/10.1504/ijmpt. $\underline{2010.031892}$ 